\title{
Wireless Network Management System for WiMAX / Wi-Fi Mesh Networks
}

\author{
Li-Der Chou, Shih-Yao Cheng, Chien-Yi Li, and Shing-Kuang Chen \\ Department of Computer Science and Information Engineering, \\ National Central University \\ No. 300, Jhongda Rd., Jhongli City, Taoyuan County 32001, Taiwan, R.O.C. \\ cldacsie.ncu.edu.tw
}

\begin{abstract}
The development of integrating several wireless network technology make wireless network devices built everywhere. In 1999, IEEE proposes WiMAX broadband wireless technology which has high transmission bandwidth and wide coverage. There are more and more researches as abovementioned wireless technology to increase Mesh network technology which can easily increase network coverage. Intel[1] point out that integrated WiMAX/ Wi-Fi Mesh network is the best solution currently. The SS of WiMAX will the bottleneck of network traffic in WiMAX/ Wi-Fi Mesh hierarchy architecture, thus we need to manage the bandwidth between WiMAX and Wi-Fi, and support Mesh network topology development.
\end{abstract}

Keywords: WiMAX, Wi-Fi, Mesh, Wireless Network, Network Management, bandwidth allocation.

\section{Introduction}

With the rapid development of wireless[2] network technologies, people look forward to experience the omnipresent internet service[3].

There are also various projects which use different technologies to build up wireless metropolitan area networks worldwide, WMAN for short. . IEEE 802.16 Working Group on Broadband Wireless Access develops WMAN that can provide high bandwidth of $70 \mathrm{Mbps}$ and long distance of $40 \mathrm{~km}$ for wireless access. According to the properties we mentioned above, WiMAX could be the solution of last-mile of home user or small and medium enterprise.

IEEE 802.16's Network Management Task Group which has been set up at 2004 aims to institute management protocol of 802.16 wireless network. IEEE $802.16 \mathrm{f}$ 2005 standard has been released at 2005 to set 802.16-2004 the MAC layer and PHY layer of fixed broadband wireless access devices and management information base (MIB), and provide 802.16 how to setup the standard of managed object. Project 802.16g draft define manager process and service, and for IEEE802.16-2004 and IEEE802.16e across fixed and mobile devices, set up standard of network management at mutual network management. Project802.16i define 802.16e MAC layer and PHY layer's MIBs of mobile broadband wireless access device. 
In WMNs deployment, each mesh node not only provides wireless service to users, but transferring packet on behalf of other nodes. Wi-Fi is already matured, and WiMAX is developing gradually. Both of them established Task groups (TGs) to specify new standard for mesh networking, such as 802.11s [4] and 802.16j [5].

Two important management functions are needed to maintain the appropriate operation of such integrated networks. The radio management used to maintain appropriate air link status, and the bandwidth management between WiMAX/ Wi-Fi mesh networks[6] are used to allocate bandwidth to Wi-Fi mesh networks connected with WiMAX network. We develop a WiMAX/ Wi-Fi mesh network management system to provide bandwidth allocation strategy among multiple Wi-Fi mesh networks based on monitoring WiMAX/ Wi-Fi mesh networks and estimating current available bandwidth on WiMAX SS.

The rest of this paper is organized as follows. In section 2, it introduces the background of WiMAX and wireless mesh network. In section 3, it proposed a hierarchical WiMAX/ Wi-Fi mesh NMS and described each module and function. Following in section 4 , an implemented system is introduced, and in section 5 , it discusses two experiments for measure bandwidth in the real network environment and measure bandwidth with bandwidth allocation and conclusions. The future works are given in section 6 .

\section{Related Work}

In 1999, IEEE 802.16 Working Group on Broadband Wireless Access start to develop standardize WMAN access interface. The development of this new standard not only stand for this technology matured but also bring lower cost of establish and network access anywhere. IEEE 802.16 standard develop going through a series of variations[7], from the begging of standard EEE 802.16 release at 2002 using 10 $66 \mathrm{GHz}$ high frequency broadband, and line-of-sight transition property, 50 kilo-meter transmission range and 72 million bits per second transmission speed at Point-to-Point mode; to improve the usage of application, IEEE 802.16 working group release 802.16a which using $2-11 \mathrm{GHz}$ and none-line-of-sight transmission property, at most 6.5 kilo-meter transmission range at Point-to-Multipoint mode; by a series of modify, IEEE 802.16-2005[8] define fixed broadband wireless access system has been release at 2004 , which define two properties smaller then $11 \mathrm{GHz}$ line-of-sight transmission system and 10-66GHz none-line-of-sight transmission system. At 2005, IEEE 802.16 release 802.16f which define Management Information. Base for fixed BWA, and define 802.16e draft-standard in order to support mobile devices.

Owing to more and more people pay close attention to this standard, Nokia and many communication companies set up WiMAX forum[9] at 2001 April. The major purpose of WiMAX forum is the orientation of IEEE 802.16, handle the communication between different brand, authentication and deal out frequency spectrum. WiMAX is regarded as one of the most important technology of broad band wireless technologies, such kind of standard is IEEE 802.16-2004 and IEEE 802.16e. Due to IEEE 802.16-2004 support fixed wireless access service, all devices which use 802.16-2004 are usually look upon to WiMAX-fixed. Now, the major development uses the property of none-line-of-sight and point-to-multi-point to would replace the 
last-mile gradually and greater than $10 \mathrm{GHz}$ to support broad bandwidth. It is suitable for network backhaul's application. And wireless devices using 802.16e usually look upon to WiMAX-mobile, supporting mobile host in WiMAX network can access WiMAX network directly.

\section{Design of Wireless Network Management System for WiMAX/ Wi-Fi Mesh Networks}

There are two assumptions in the WiMAX/ Wi-Fi mesh NMS. At first, all of the WiMAX/ Wi-Fi mesh devices need SNMP supporting; and the other assumption is that the devices must support ICMP PING or we need to know the IP address of WiMAX BS.

This paper proposed the WiMAX/ Wi-Fi mesh NMS network management architecture as Figure 1; it has two major tasks which are described as follows:

Firstly, the Wi-Fi mesh NMS manages Wi-Fi APs and Wi-Fi mesh APs. The WiMAX mesh NMS not only manages WiMAX BS and SS, but also collects statistic information from Wi-Fi mesh NMS. Secondly, WiMAX/ Wi-Fi mesh NMS update the data of WiMAX/ Wi-Fi mesh devices constantly.

The system analyses from the user point are divided into two parts: one is from the client side and the other is from the server side. The client side only needs JAVA language supporting. And the server side includes the management system that provides all functions and services; the design of server side is divided into six parts as follows: Graphical User Interface (GUI) module, Authentication module, Discovery module, Performance module, Monitor module, Bandwidth module.

The section introduces the functions of each module on WiMAX/ Wi-Fi mesh NMS. It also presents these functionalities and usages that can be achieved through the proposed architecture in each module. All of these functionalities work with devices which must have SNMP agent supporting.

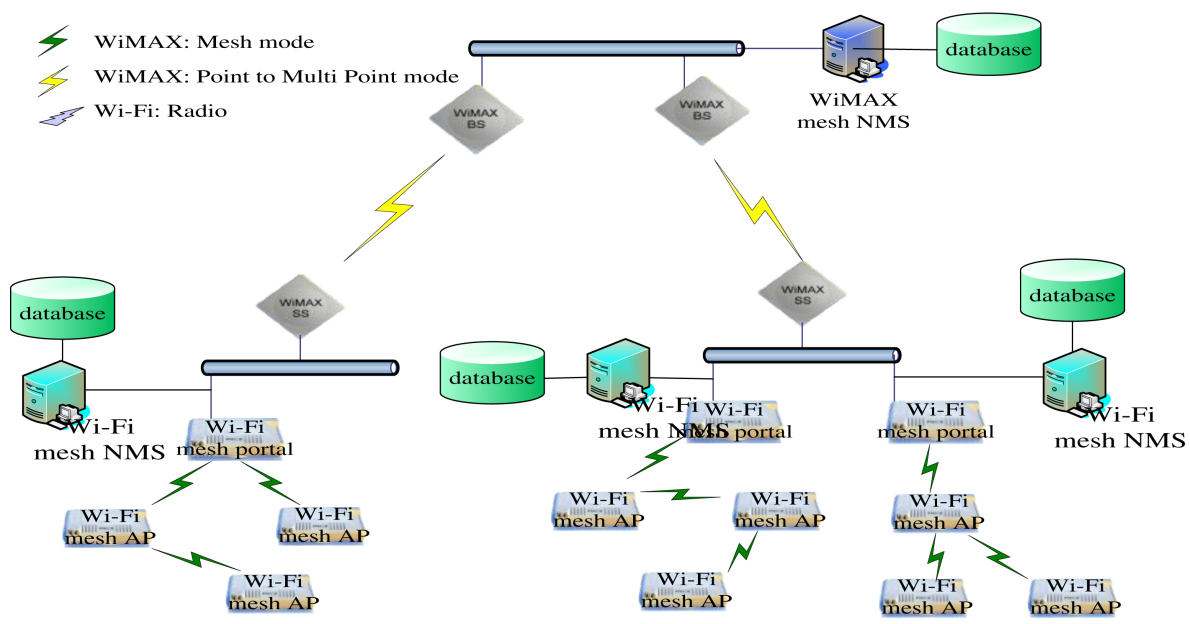

Fig. 1. Architecture of WiMAX/ Wi-Fi mesh network management system 
(1) Functions of GUI module

Device topology: this function provides the physical mapping of the discovered devices. It shows as the hierarchical tree architecture. It is convenient for managers to see the relationship of whole network. Discovery request: the manager could input a range of IP address or input an IP address for WiMAX $\mathrm{BS}$, and then the GUI module would send the request to Discovery module. Change modulation type: the managers can change modulation type on WiMAX BS and SS on demand, the WiMAX/ Wi-Fi mesh NMS supports 7 different modulation types such as BPSK 1/2, QPSK 1/2, QPSK 3/4, 16QAM 1/2, 16QAM 3/4, 64QAM 2/3 and 64QAM 3/4. Power selection: the managers can use this function to change the power level of interfaces on Wi-Fi mesh AP individually. The Wi-Fi NMS implements four power levels such as 100\%, 50\%, $25 \%$ and $12.5 \%$. Interface selection: the managers can use this function to select which interface to monitored or controlled.

(2) Functions of authorization module

Authentication manager log: this function provides the history manager logs that manager login time and logout time. Manager action log: this function provides the history manager action logs that records the manager behaviors during the using time.

(3) Functions of discovery module

Wi-Fi AP: this function can discover Wi-Fi devices in the managed domain. Wi-Fi mesh AP: this function can discover Wi-Fi mesh AP in the managed domain. WiMAX BS: this function can discover WiMAX BS in the managed domain, and find out the SSs connected with BS. WiMAX SS: this function can discover WiMAX SS in the managed domain.

Functions of performance module:

Input statistics: the function provides the input traffic report. Output statistics: the function provides the output traffic report. Probability density function: the function provides the p.d.f report. Cumulative distribute function: the function provides the c.d.f report.

(4) Functions of monitor module

WiMAX monitoring: the function provides the ability to monitor WiMAX MIB, which could monitor WiMAX SS directly or via WiMAX BS. Wi-Fi monitoring: the function provides the ability to monitor Wi-Fi MIB. Wi-Fi mesh monitoring: the function provides the ability to monitor a Wi-Fi mesh networks in hierarchical way.

(5) Functions of bandwidth module:

Estimated bandwidth[10][11]: the function could estimate current bandwidth on WiMAX SS while SS changed modulation type. Strategy[12]: the function compiles bandwidth allocation strategy for multiple Wi-Fi mesh networks connected with a WiMAX SS. There are two strategies in the system. The first one is "static" which allocate bandwidth by SS table written by estimated bandwidth function. The other one is "percentage" which allocates bandwidth is according with the need of available and request. Active: the function sends bandwidth allocation strategy to WiMAX SS and actives bandwidth allocation function on SS. 


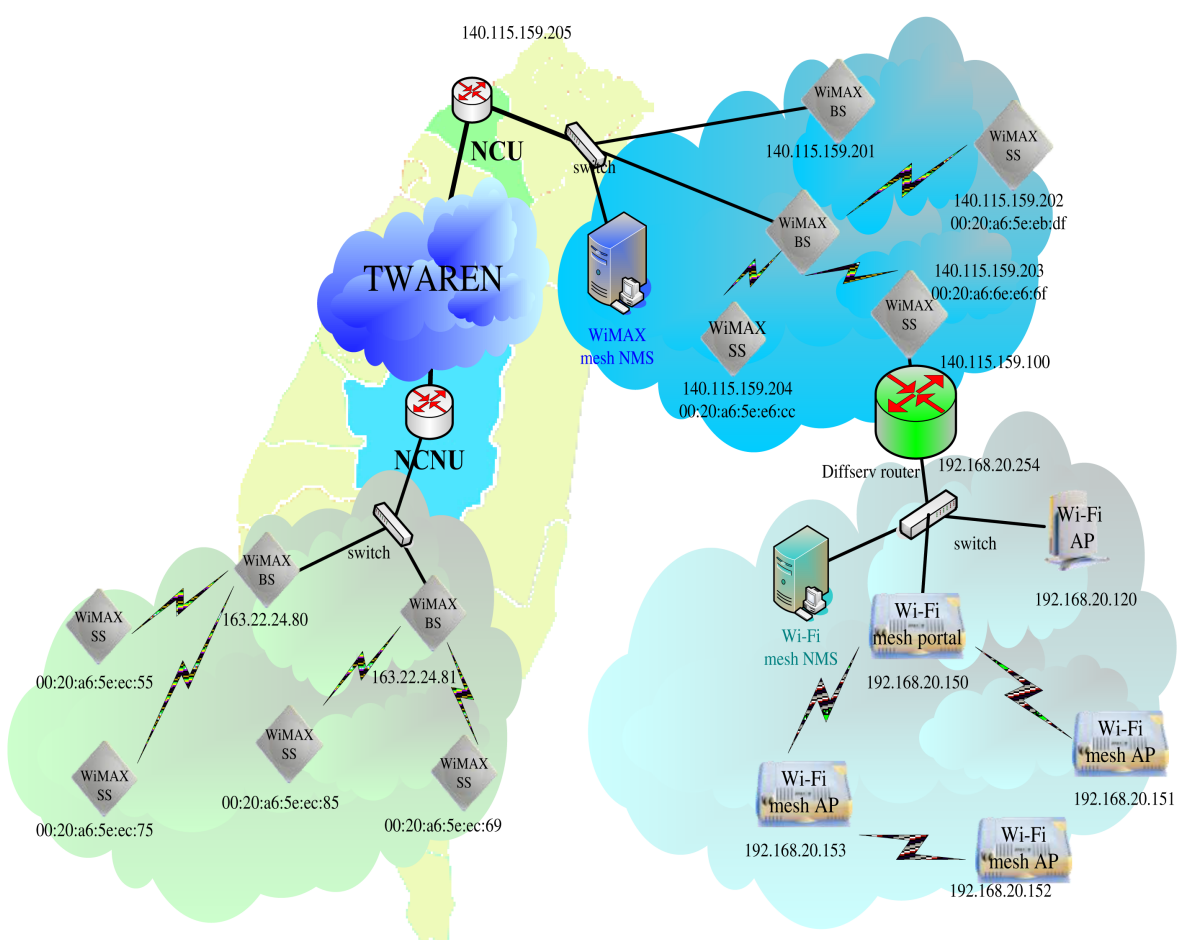

Fig. 2. Implementation environment

\section{Implementation Environment and Develop Tools}

It becomes more important issue that needs to be solved is how to integrate and manage the different wireless networks which includes WiMAX/ Wi-Fi mesh networks efficiently. In 2005, NCU and NCNU established a WiMAX networks test environment, and we plus a Wi-Fi mesh network connected with the NCU WiMAX SS. Figure 2 shows the deployment environment. In NCU, there are two Proxim MP16 $3500[13] \mathrm{BSs}$ installed at the rooftop of the Library, each equipped a $60^{\circ}$ antenna. The Proxim MP16 3500 SSs are installed at the rooftop of the electrical engineering building, communication center and lefting one for portable test. In NCNU, there are also two Proxim MP16 3500 BSs and four Proxim MP16 3500 SSs be installed in the campus. We implement the WiMAX/ Wi-Fi mesh NMS for the networks which could be divided into two parts: At first, WiMAX mesh NMS is set in the library of the NCU which are used to manage WiMAX networks in NCU and NCNU, and the Wi-Fi mesh NMS is setting in the lab which are used to manage the private Wi-Fi mesh networks.

We use the Windows XP SP2 as the development and implementation platform. The develop environment that we need is to install JDK 1.5.0 which makes JAVA program executable, then we install Apache as the WEB server which is the most popular WEB server currently and Tomcat for execute JSP program, and MySQL as database server. Table 1 show several tools utilized during the developing process. 
We use the JAVA language to build the kernel of most modules and Java Server Pages (JSP), JAVA Script and JAVA Applet to build a familiar GUI for managers. All of them can be executed in all kinds of operation systems and all develop tools are open source. It also uses the free application programming interface (API) of Westhawk's Java SNMP stack 4_13 to implement SNMP operation for requesting the data set of WiMAX/ Wi-Fi mesh devices, and use MG-SOFT Browser to verify the SNMP operation be executed correctly in the proposed system.

The implementation of the proposed system could be divided into two parts, such as WiMAX mesh NMS and Wi-Fi mesh NMS. WiMAX mesh NMS could management the WiMAX mesh devices and get information from Wi-Fi mesh devices by connecting to Wi-Fi mesh NMS. We have a web-based GUI used to communicate with manager, who log into the system by verifying name and password. After verifying, the system shows main page on web browser, and it be divided into three frames. At first, the top frame could discover the WiMAX devices on WiMAX mesh NMS by input a range of IP addresses or adding IP address for known WiMAX BS, and discover the Wi-Fi mesh devices on Wi-Fi mesh NMS, then monitor the module would find out the WiMAX SSs connected with the BS automatically. Secondly, the right frame shows the topology of the WiMAX/ Wi-Fi mesh network on the WiMAX mesh NMS. Finally the left frame shows the discovery result, list of the managed devices and the advanced information for configuring WiMAX/ Wi-Fi mesh devices, the function of power selection are implemented with interfaces on Wi-Fi mesh AP and the function of change modulation type are implemented on WiMAX devices.

\section{Experiments and Discussions}

Experiment results and discussions are presented in this chapter. The experimental environment as shown in Figure 3, the experiment adopts "static" bandwidth allocation strategy for endpoint 2 for uplink takes $512 \mathrm{Kbps}$ of estimated bandwidth and downlink takes $1024 \mathrm{kbps}$ of estimated bandwidth and adopts "percentage" bandwidth allocation strategy for endpoint 3 for uplink takes $10 \%$ of estimated bandwidth and downlink takes $20 \%$ of estimated bandwidth. The traffic generator generates traffic between endpoint 1 and endpoint 2, endpoint 3, and record the result for discussion. We changed modulation types for testing if the system could allocate bandwidth to different Wi-Fi mesh networks connect with the same WiMAX SS according to the bandwidth allocation strategy for each Wi-Fi mesh network.

Table 1. Develop tools

\begin{tabular}{|c|l|}
\hline Develop tools & \multicolumn{1}{|c|}{ Name and Version } \\
\hline Language & JAVA, JSP 2.0, JAVA Script \\
\hline Programming & Eclipse V.3.0.1 \\
Platform & Macromedia Dreamwaver MX \\
\hline SNMP agent & Westhawk's Java SNMP stack \\
& 4 13 \\
\hline MIB browser & $\begin{array}{l}\text { MG-SOFT Browser Edition } \\
\end{array}$ \\
& 7.10 .0 .3880 \\
\hline
\end{tabular}


Table 2. Result of experiment 1

\begin{tabular}{|c|c|c|c|c|}
\hline Modulation & $\begin{array}{c}\text { Mean } \\
\text { bandwidth in } \\
\text { uplink }\end{array}$ & $\begin{array}{c}\text { Mean } \\
\text { bandwidth in } \\
\text { downlink }\end{array}$ & $\begin{array}{c}\text { Standard } \\
\text { deviation in } \\
\text { uplink }\end{array}$ & $\begin{array}{c}\text { Standard } \\
\text { deviation in } \\
\text { downlink }\end{array}$ \\
\hline 64QAM3/4 & 500 & 1149 & 0.05779 & 0.131916 \\
\hline 42QAM2/3 & 426 & 834 & 0.03167 & 0.09671 \\
\hline 16QAM3/4 & 505 & 1138 & 0.04977 & 0.15817 \\
\hline 16QAM1/2 & 505 & 1070 & 0.06193 & 0.15908 \\
\hline QPSK3/4 & 522 & 876 & 0.03873 & 0.21234 \\
\hline QPSK1/2 & 505 & 633 & 0.06757 & 0.19479 \\
\hline BPSK1/2 & 391 & 352 & 0.04452 & 0.03355 \\
\hline
\end{tabular}

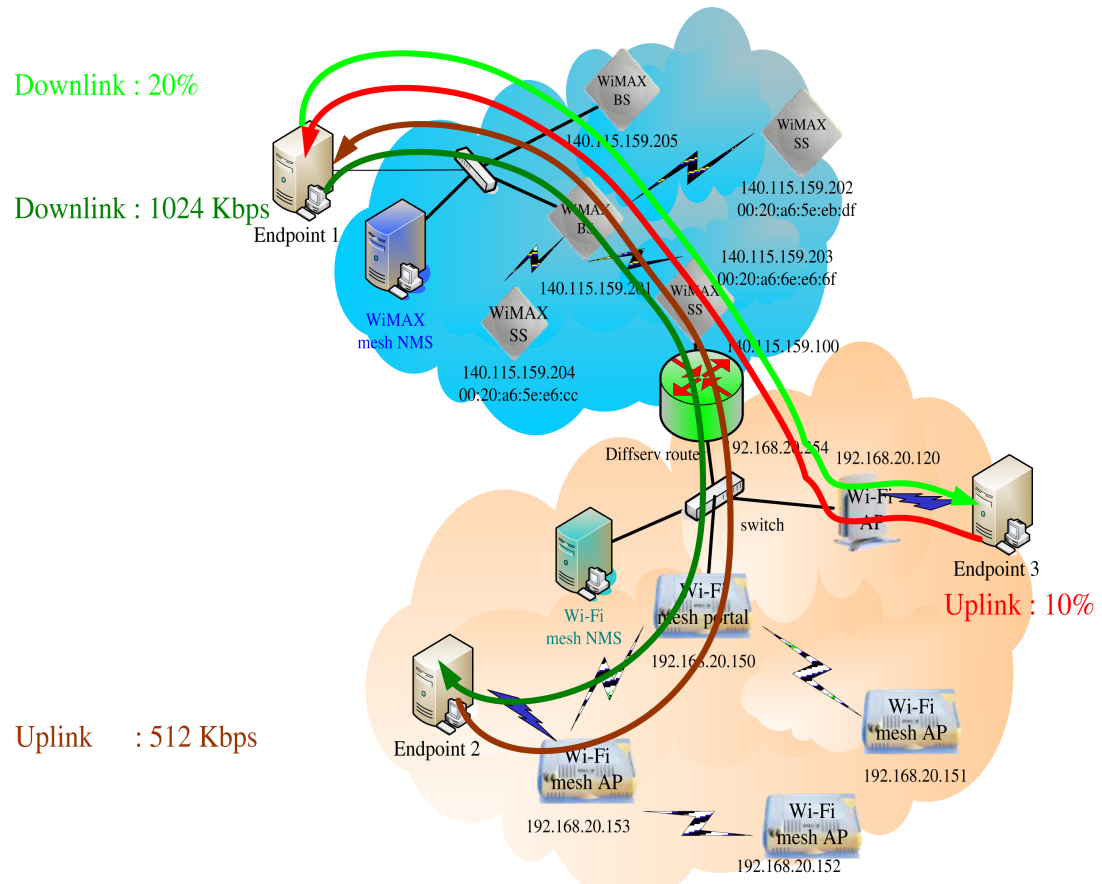

Fig. 3. Experimental environment

Objective: This experiment compares with "static" and "percentage" bandwidth allocation strategies for Wi-Fi mesh networks connected with WiMAX SS. With the monitor of WiMAX SS, the WiMAX/ Wi-Fi mesh NMS detected the modulation change and use associated bandwidth allocation strategies to reallocate bandwidth for multiple Wi-Fi mesh networks. Method: All of the endpoints execute endpoint program, then start throughput test in BPSK 1/2, QPSK 1/2, QPSK 3/4, 16QAM 1/2, 16QAM 3/4, 64QAM 2/3 and 64QAM 3/4 modulation for uplink and downlink 
direction. Experiments results and discussions: The experiment1 throughputs for Endpoint 2, which be guaranteed static downlink 1024 Kbps and uplink 512 Kbps.

Table 3. Result of experiment 2

\begin{tabular}{|l|l|l|l|l|}
\hline Modulation & $\begin{array}{l}\text { Mean } \\
\text { bandwidth in } \\
\text { uplink }\end{array}$ & $\begin{array}{l}\text { Mean } \\
\text { bandwidth in } \\
\text { downlink }\end{array}$ & $\begin{array}{l}\text { Standard } \\
\text { deviation in } \\
\text { uplink }\end{array}$ & $\begin{array}{l}\text { Standard } \\
\text { deviation in } \\
\text { downlink }\end{array}$ \\
\hline 64QAM3/4 & 562 & 1211 & 0.04067 & 0.11603 \\
\hline 42QAM2/3 & 484 & 1088 & 0.03167 & 0.09135 \\
\hline 16QAM3/4 & 491 & 843 & 0.03562 & 0.05891 \\
\hline 16QAM1/2 & 243 & 547 & 0.01683 & 0.06280 \\
\hline QPSK3/4 & 184 & 412 & 0.01639 & 0.05504 \\
\hline QPSK1/2 & 125 & 273 & 0.0086 & 0.03753 \\
\hline BPSK1/2 & 57 & 128 & 0.00673 & 0.02304 \\
\hline
\end{tabular}

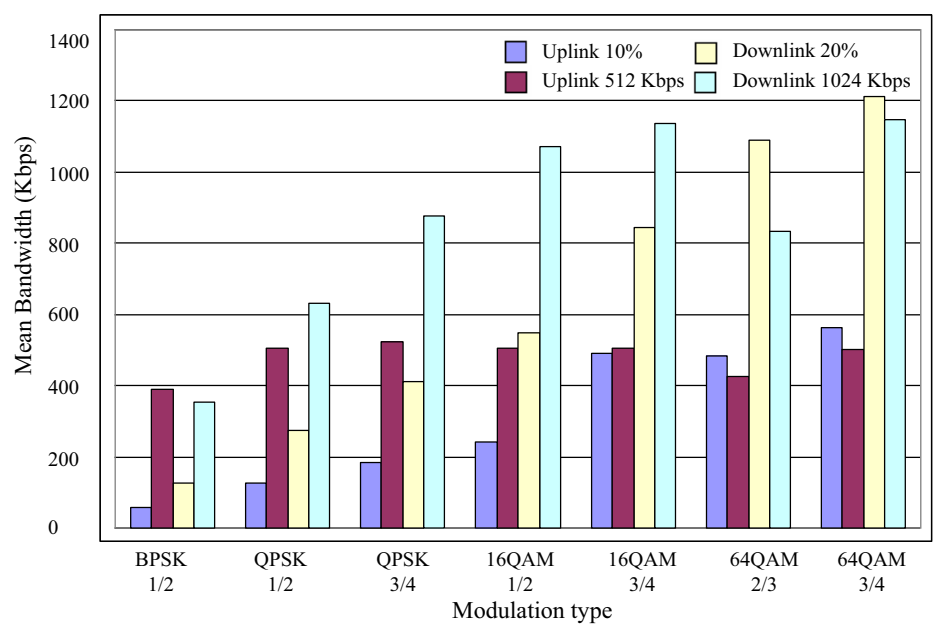

Fig. 4. Mean bandwidth for uplink and downlink with percentage BW allocation strategy in different modulation type

The same situation in experiment 2 occurs on uplink while the modulation is less complex than QPSK 1/2. The total bandwidth could not provide enough bandwidth to guarantee the bandwidth agreement. Then the experiment use "percentage" bandwidth allocation policy which is guaranteed $20 \%$ of estimated downlink bandwidth and $10 \%$ of estimated uplink bandwidth, the experiment proceeded between Endpoint1 and Endpoint3.

We discuss the static and percentage bandwidth allocation strategy in Figure 4, the Wi-Fi mesh network takes less bandwidth by percentage bandwidth allocation strategy when the modulation type less complex than 16QAM 3/4, from the result, the static bandwidth allocation strategy almost allocate much bandwidth to a Wi-Fi mesh 


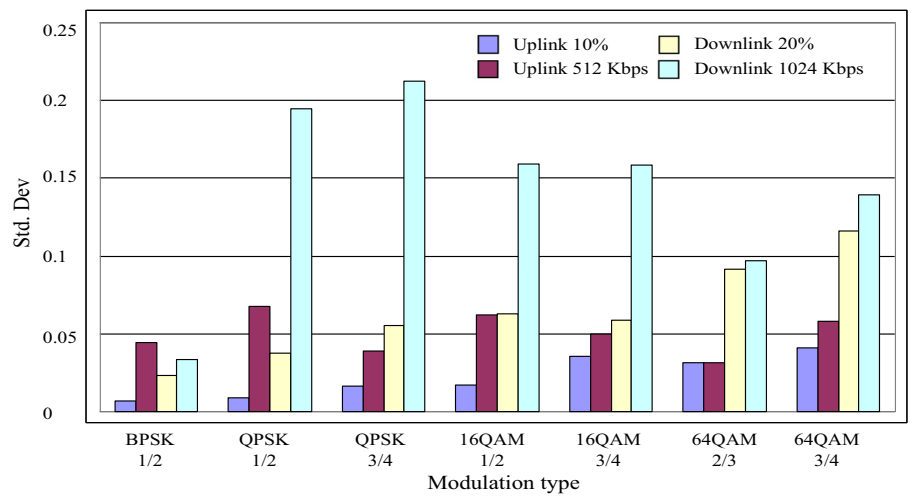

Fig. 5. Standard deviation for uplink and downlink with percentage BW allocation strategy with different modulation type

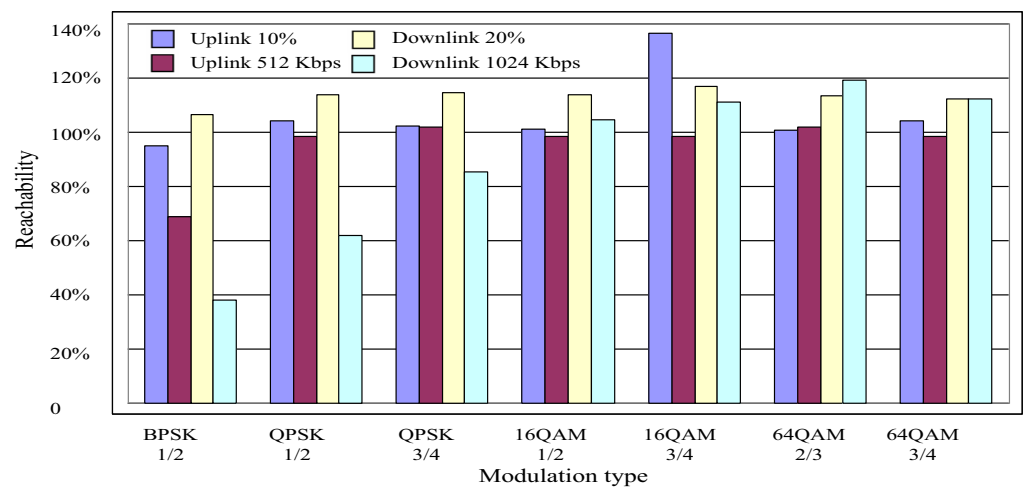

Fig. 6. Reachability for percentage and static bandwidth allocation strategies

networks, however, in Figure 5 shows that it has large standard deviation, which affects the stable service such as VoIP, the percentage allocation policy seems work well in each modulation type, but the guaranteed bandwidth variation large based on modulation type changed, we make a series of experiments with different modulation types for verification. After all, we compares the reach rate for static bandwidth allocation strategy and percentage bandwidth allocation strategy with their expected value, as Figure 6 shows, the reach rate might more than $100 \%$, that is caused by bandwidth management capability of CBQ. In percentage bandwidth allocation strategy, it always keeps reach rate up than $95 \%$, but in static bandwidth allocation strategy, the range of reach rate varies from $38 \%$ to $119 \%$, even the Wi-Fi mesh NMS get the information of SS, it could not estimated the bandwidth he would get correctly. Considering of this situation, the percentage bandwidth allocation strategy works better. 


\section{Conclusions}

In this paper, first of all we explain the importance of WiMAX/ Wi-Fi mesh network management system in the WiMAX/ Wi-Fi mesh networks, and introduce the architecture of our proposed WiMAX/ Wi-Fi mesh network management system. Monitor module and bandwidth module, which could monitor the WiMAX/ Wi-Fi mesh network status and reallocate bandwidth to multiple Wi-Fi mesh networks connected with a WiMAX SS accordingly. We provide "static" and "percentage" bandwidth allocation schemes, in static way, the mesh network are always promised a static bandwidth, it is more less complexity than percentage way, the "percentage" bandwidth allocation scheme provides high flexibility but increase system load by reallocating in each modulation type changed, the two kinds of bandwidth allocation schemes could be apply for different service need.

\section{Acknowledgments}

This research was supported in part by National Science Council of the Republic of China under contracts NSC 93-2219-E-260-006, NSC 95-2627-E-008-002 and NSC 95-2221-E-008-031.

\section{References}

1. Intel Corp., Understanding Wi-Fi and WiMAX as Metro-Access Solution (2004), http://www.intel.com/netcomms/technologies/wimax/304471.pdf

2. Chou, L.-D., Lu, C.-C., Lu, C.-Y.: Design of location management for heterogeneous wireless networks. LNCS. Springer, Heidelberg (to appear, 2007)

3. Chou, L.-D., Chen, J.-M., Kao, H.-S., Wu, S.-F., Lai, W.: Seamless streaming media for heterogeneous mobile networks. ACM Springer Mobile Networks and Applications 11(6), 873-887 (2006)

4. Hauser, J.: Draft PAR for IEEE 802.11 ESS Mesh, IEEE Document Number: IEEE 802.11-03/759r2

5. IEEE 802.16's Mobile Multihop Relay (MMR) Study Group, http://ieee802.org/16/sg/mmr/

6. Chou, L.-D., Hsieh, H.-J., Chen, J.-M.: Multicast with QoS support in heterogeneous wireless networks. In: Yang, L.T., Guo, M., Gao, G.R., Jha, N.K. (eds.) EUC 2004. LNCS, vol. 3207, pp. 581-590. Springer, Heidelberg (2004)

7. Eklund, C., Marks, R.B., Standwood, K.L., Wang, S.: IEEE Standard 802.16: A Technical Overview of the WirelessManTM Air Interface for Broadband Wireless Access. IEEE Communications Magazine, pp. 98-107 (June 2002)

8. Marks, R.B., Stanwood, K., Chang, D.n.: IEEE Standard for Local and Metropolitan Area Networks Part 16: Air Interface for Fixed Broadband Wireless Access Systems, IEEE Standard (October 2004)

9. WiMAX Forum, http://www.wimaxforum.org 
10. Chen, Y.W.: Traffic Behavior Analysis and Modeling of Sub-networks. International Journal of Network Management 12(5), 323-330 (2002)

11. Chen, Y.W., Chou, C.-C.: Correlation based Traffic Modeling of Sub-networks. Journal of Internet Technology

12. Chen, Y.W., Hu, S.-H.: Study of the Traffic Scheduler by Using Correlation Heuristics. IEICE Trans. on Communications, 2273-2280 (2004)

13. Proxim Wireless Achieves WiMAX Forum Certification for Tsunami ${ }^{\circledR}$ MP.16 3500 Product, http://www.proxim.com/products/bwa/multipoint/16_3500/ index.html 